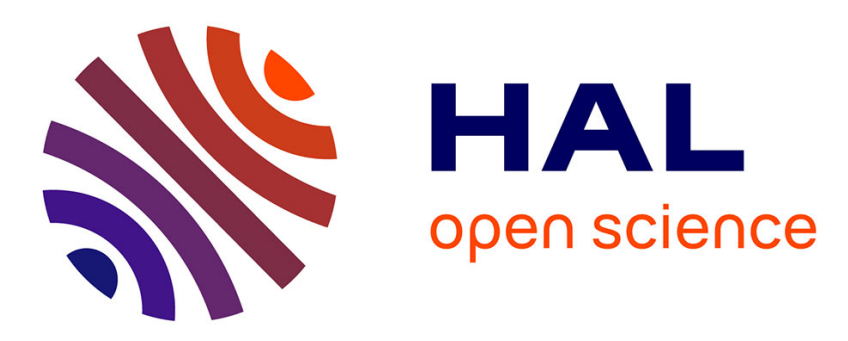

\title{
Random 3D-printed isotropic composites with high volume fraction of pore-like polydisperse inclusions and near-optimal elastic stiffness
}

\author{
M.G. G Tarantino, O. Zerhouni, K. Danas
}

\section{- To cite this version:}

M.G. G Tarantino, O. Zerhouni, K. Danas. Random 3D-printed isotropic composites with high volume fraction of pore-like polydisperse inclusions and near-optimal elastic stiffness. Acta Materialia, 2019, 175, pp.331-340. 10.1016/j.actamat.2019.06.020 . hal-02167837

HAL Id: hal-02167837

https://hal-polytechnique.archives-ouvertes.fr/hal-02167837

Submitted on 28 Jun 2019

HAL is a multi-disciplinary open access archive for the deposit and dissemination of scientific research documents, whether they are published or not. The documents may come from teaching and research institutions in France or abroad, or from public or private research centers.
L'archive ouverte pluridisciplinaire HAL, est destinée au dépôt et à la diffusion de documents scientifiques de niveau recherche, publiés ou non, émanant des établissements d'enseignement et de recherche français ou étrangers, des laboratoires publics ou privés. 


\title{
Random 3D-printed isotropic composites with high volume fraction of pore-like
}

\section{polydisperse inclusions and near-optimal elastic stiffness}

\author{
M. G. Tarantino, O. Zerhouni and K. Danas*
}

LMS, CNRS, Ecole Polytechnique, Institut Polytechnique de Paris, Palaiseau, 91128,

France

Keywords: Closed-cell Porous material, Bulk and Shear moduli, finite element modeling, Homogenization.

\begin{abstract}
Highly porous materials with random closed-cell architecture combine isotropy with high stiffness. Yet in practice, the complexity of their manufacturing limits the experimental exploration of these materials, for which studies of the elastic response remain to date mainly theoretical. In this study, we measure experimentally the elastic moduli of random closed-cell porous-like composites fabricated by 3Dprinting. These materials contain a high volume fraction (up to 82 vol pct) of nonoverlapping, polydisperse void-like spherical inclusions, which are randomly dispersed in a homogeneous polymer matrix. We first generate the virtual microstructures of these materials using a random sequential adsorption (RSA) algorithm, and then use numerical homogenization to compute the size of the material representative volume element (RVE). The latter is used to assemble the test samples, whereby the void-like inclusions are 3D-printed using a gel-like polymer with mechanical properties that are in high contrast with those of the base polymer thus behaving mechanically as pores. Experiments reveal that the proposed isotropic
\end{abstract}

\footnotetext{
*Corresponding author (konstantinos.danas@polytechnique.edu)

Published in Acta Materialia 175 (2019), 331-340, https://doi.org/10.1016/j.actamat.2019.06.020
} 
random closed-cell porous materials have bulk and shear moduli that lie very close to the theoretical Hashin-Shtrikman upper bounds for an isotropic porous solid.

\section{Introduction}

Over the past two decades, rationally and virtually designed (meta-)materials have revolutionized our ability to exploit optimized architectures to achieve unprecedented, and if possible optimal, material properties. Examples are numerous and span several fields, from electro-magnetism [1],[2] and optics [3] to acoustics [4], mechanics [5], [6] and, more recently, mechanobiology [7], [8]. One of the major elements that promoted the rapid proliferation of such materials, is the advent of additive manufacturing. Using 3D laser lithography for example, systems with oddly shaped architectural features can be fabricated with design control down to only a few nanometers [9]. Equally important, the versatility of current 3D printing technologies enables the realization of composite architectures that cannot be fabricated in any other way, e.g., wood-inspired fiber-reinforced honeycombs [10] and two-phase cocontinuous solids that mimic biological exoskeletons and block copolymers [11]. This ability to incorporate complex architectures into a material system, coupled with the robustness of modern computational methods, has enabled extending the portfolio of materials now available to scientists and engineers.

The most ubiquitous case study among architected materials is that of cellular solids, whereby an interconnected network of solid struts or plates forms the edges or faces of the cells [12]. Cellular solids are high-porosity materials with either periodic or stochastic architecture. In nature, as in most of synthetic structural materials, cellular solids have a three-dimensional (3D) architecture that typically consists of a 
random packing of polyhedron cells. Stochastic foams, as these materials are also called, have been a topic of intense research prior to the advent of metamaterials [13][19]. Over the past two decades, highly porous metal "sponges" were extensively produced using the replication processing [20], a method developed by Mortensen and Fitzgerlad in the late 90s [21]. In recent years, however, these materials have attracted far lesser attention than cellular solids with a periodic architecture (mainly lattices). The reason for this is two-fold. First, periodic cellular materials exhibit higher strength and stiffness than stochastic foams of the same density because of the higher strain-energy stored during deformation. The latter is governed by cell wall stretching rather than bending [22]. Second, lattices are also naturally amenable to optimization and thus offer unparalleled flexibility in achieving topology-optimized architectures depending, for instance, on the design objective, constituent material and manufacturing method. Noteworthy is the octet truss lattice by Deshpande et al. [23]. By virtue of its ideal nearly linear scaling of mechanical properties with density, such cellular systems have paved the way to novel lattice architectures with maximized strength and stiffness per unit weight. Examples comprise the ultra-low density lattices of high-strength metals and ceramics, whereby the cells are composed of either hollow trusses [24]-[28] and continuous thin shells [29] ("shellular") with dimensions extending from nanometers to microns. These systems efficiently combine the structural advantages of their stretch-dominated cellular geometry with the strengthening size effects of their nano- or microscale features, and are today the lightest, stiffest and strongest metamaterials achieved. However, the poor scalability and highly anisotropic response largely limit the use of such cellular materials for macroscale engineering applications. 
From a different viewpoint, there exists a large body of theoretical work in the context of homogenization [30]-[31] dealing with the optimal elastic properties (stiffness) of composite materials. In such theoretical studies, and specializing the discussion to porous materials, it has been known for long time that various microstructures may attain the optimal Hashin-Shtrikman (HS) theoretical bound for the bulk and/or shear modulus [32], [33]. Notable examples include the Hashin composite sphere assemblage (CSA) for the bulk modulus [34] and the high-rank laminates [35] among others [36], [37]. However, despite recent advances in current manufacturing technologies, the fabrication of those theoretical microstructures remains very difficult (if not impossible), as they usually comprise a very large (even infinite) number of scales. In the present study, we take inspiration from these seminal homogenization studies to realize porous-like microstructures with near-optimal elastic moduli.

In very recent years, two novel closed-cell lattices, composed of plates rather than beams or shells, have emerged as promising candidates for approaching the theoretical limit of isotropic elastic stiffness. These are the cubic-octet foam proposed by Berger et al [38] and the plate-lattice designed by Tancogne-Dejean et al [39]. The first consists of a combination of two cellular geometries, i.e., a cubic and an octet foam. The second is obtained by placing plates along the closest-packed planes of crystal structures with cubic symmetry. Unlike open-cell truss lattices, closed-cell plate-lattices involve in-plane deformation of their constituent plates and thus better utilize material volume at different loading directions. The potential impact of these two new metamaterials has been mainly shown numerically by a purely linear elastic analysis, ignoring any local nonlinear effects due to stress and strain concentrations. In addition, these studies lack a thorough experimental demonstration except in a 
limited number of cases [39]. In particular, due to the closed-cell geometry and current limitations in the maximum inclination angle of 3D printing technology, one is unable to remove (except only partially) the internal non-welded metallic powder or support material in polymers, thus making the use of relative density to analyze such materials unsuitable.

In this work, we propose an alternative route to realize isotropic multi-scale closed-cell porous materials by taking inspiration from seminal studies on homogenization of two-phase composites [33],[34] containing random distribution of non-overlapping spherical inclusions/voids [40]-[43]. Specifically, we examine the elastic response of porous-like solids consisting of non-overlapping, finite polydisperse (i.e. multiple size) spherical inclusions that are randomly dispersed into a homogeneous matrix. Those inclusions are made of a very soft support material, which has a Young's and bulk modulus that is one thousand times smaller than that of the matrix phase but of similar density. This result, which is discussed further in the following, allows treating such materials as "porous-like" composites in terms of their quasi-static mechanical response given the very high inclusion/matrix contrast, but not as cellular solids in terms of relative density since the support material has (almost) the same density of the matrix. Henceforth, the term "porosity", denoted with $c$, refers to the volume fraction of the pore-like inclusions such that $1-c$ denotes the remaining volume fraction of the matrix phase (and not the relative density like for cellular solids and foams).

In particular, we exploit the polydispersity of the spherical voids to generate random porous architectures that provide an experimentally feasible approximation of the Hashin CSA model-microstructure [34]. This theoretical model is known to achieve the theoretical limit of isotropic elastic compressibility but inherently 
involves an "infinite" range of length scales, which makes the manufacturing of such composites practically impossible to achieve. To overcome this issue, we develop a numerical protocol that enables generating RVEs of multi-inclusion material systems, whereby high volume fractions of spherical voids are obtained by employing inclusions with very different diameter. The virtual realizations of such heterogeneous materials are then transformed into physical microstructures via the use of a $3 \mathrm{D}$ polymer printer. Using relatively large computational resources, we are able to explore experimentally and numerically a very large range of porosities that spans values from 0 to 0.82 . In particular, we show that our 3D-printed random porous architectures are almost isotropic (both experimentally and numerically) and yield values of the effective elastic moduli that lie near the corresponding HashinShtrikman upper bounds.

\section{Methods}

\subsection{A modified RSA algorithm for the generation of multi-inclusion systems}

Virtual realizations of the porous microstructures containing finite polydisperse spherical voids are obtained using a random sequential addition (RSA) procedure. This consists in introducing randomly, irreversibly and sequentially non-overlapping objects of arbitrary shape and size (here spherical inclusions) into a cubic cell [40][43]. The algorithm is highly versatile, and to date it has been used to generate systems containing random distributions of monodisperse (i.e. single sized) [42], [44] and polydisperse spheres [43], and more recently also mono- and polydisperse ellipsoids of arbitrary aspect ratios and orientations [45]. Polydisperse spherical inclusions are generated from the inclusion center and diameter $D_{i}$, and those intersecting the cell outer surfaces are cut off and copied to the opposite face of the 
cube. The generated cubic unit cells are periodic, and contain a finite number of families of identical spheres (here pores) randomly dispersed in the cell volume. In Figure 1a, we show a few representative RSA-generated cubic cells containing a volume fraction of spherical pores, thereinafter denoted as $c$, which ranges from 0.2 to
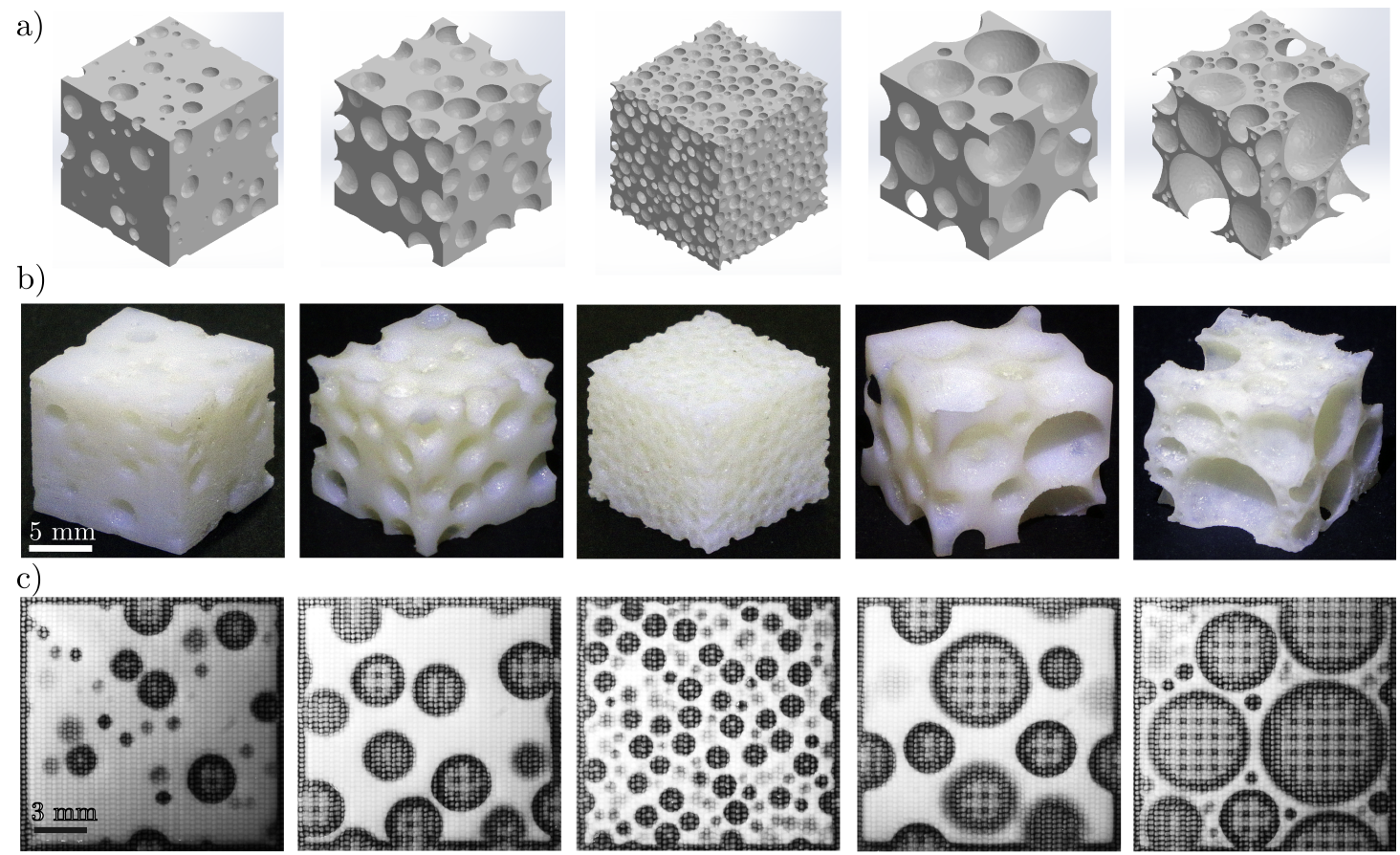

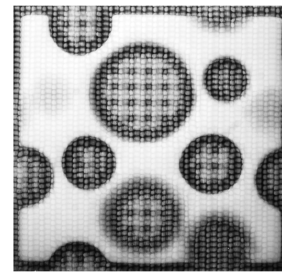

0.6

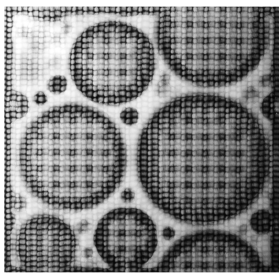

0.75

Figure 1: (a) RSA-generated periodic unit cells of multi-inclusion systems containing a random distribution of spherical voids with different size at various volume fractions. (b) Optical images of the corresponding 3D-printed unit cells. (c) Micrographs of the 3D-printed porous microstructures obtained upon interrupting the 3D printing process at a build volume of thickness $\sim 500 \mu \mathrm{m}$. The porelike inclusions are built by the 3D-printer using a gel-like support material (i.e. nearly transparent phase). Pores through thickness are also visible (i.e. light grey phase).

0.75 .

In order to achieve such high volume fractions of inclusions, especially up to 0.82 , we need to modify the RSA algorithm adopted in Refs.[43]-[44]. Specifically, the modified RSA algorithm (which is described in detail in Refs.[43]-[44]) takes as input only two parameters, i.e. the diameter of the largest spherical pore family, i.e. $D_{\max }$, and the desired inclusion volume fraction (i.e. the porosity $c$ ).

Following the standard RSA process, the algorithm starts by generating pores with diameter $D_{\max }$ until no additional void of that family can be further inserted without 
overlapping with any of the previously accepted voids. The cutoff value of iterations for moving to the next void family is set to 3000 consecutive rejected inclusion positions. Next, the algorithm reduces the size $D_{i}$ of the new generated pore family by $1 \%$. At each step, a minimum distance between any two neighboring inclusions is imposed and the periodicity on opposite faces is enforced. In this study, the minimum thickness of the matrix ligament between two inclusions is set equal to $\sim 150 \mu \mathrm{m}$, which corresponds to the best resolution of our 3D-printer (see discussion in Section 2.3). This procedure continues until the desired porosity $c$ is reached. We note in passing that due to the sequential addition of voids the target porosity is reached up to a small deviation, which simply corresponds to the volume ratio between the last (and smallest) inclusion and that of the entire cell. The generated virtual microstructures are fabricated by 3D printing (see Figure $1 \mathrm{~b}, \mathrm{c}$ ). This imposes an additional geometric constraint in the RSA process, which is the minimum attainable pore diameter, denoted as $D_{\text {lim }}$ (see discussion in Section 2.3). In this study, we set $D_{\text {lim }}=250 \mu \mathrm{m}$, which is the smallest pore size experimentally attainable by our 3D-printer.

\subsection{Finite Element Modeling}

Numerical estimates of the homogenized elastic moduli of the random porous microstructures are obtained by linear elastic finite element (FE) analysis carried out with Abaqus. Simulations are performed using standard quadratic 10-node tetrahedral isoparametric elements (i.e. C3D10 in Abaqus notation). An isotropic linear elastic material model is used for modelling the matrix phase and the elastic moduli of the constituent polymer are measured experimentally during tensile multi-step relaxation testing (see Section 2.3). Throughout the numerical study, and given the very low elastic moduli of the gel-like material, the simulations are carried out assuming that the spherical inclusions are voids. Mesh-refined FE models of the porous 
microstructures are generated using the automatic mesh generator NETGEN [46]. The converged RVEs comprise approximately $1.2 \mathrm{M}$ degrees of freedom (d.o.f.) for the lower porosities $(c<0.1), 5 \mathrm{M}$ d.o.f. for the moderate ones $(0.3<\mathrm{c}<0.5)$ and up to $18 \mathrm{M}$ d.o.f. for the highest porosity $(c=0.82)$. Moreover, special care is taken to ensure pairing of the nodes on opposite faces of the cubic cell, which is necessary for the application of the periodic boundary conditions discussed in the following section.

\subsubsection{Protocol for the determination of converged RVEs for experiments}

The very first step towards the experimental realization of the proposed microstructures is the determination of the RVE physical dimensions that enable meaningful measurements of the effective elastic moduli in experiments, where the applied boundary conditions are mixed [44]. Specifically, our first goal is to determine, by means of numerical homogenization and for every given porosity, the geometrical RVE that yields values of the elastic constants independent on the applied boundary conditions. To this end, prior to experimental testing, we carry out an extensive computational investigation where cubic unit-cells, containing a given volume fraction of polydisperse pores, are subjected to both periodic boundary conditions (PBC) and kinematically uniform boundary conditions (KUBC) (see details in Ref. [47]). Specifically, these boundary conditions are implemented in a similar fashion to Ref. [48], and notably are defined such that the displacement field $\boldsymbol{u}(\boldsymbol{x})$ at point $\boldsymbol{x}$ in the microstructure is given by

$$
(\mathrm{PBC}): \boldsymbol{u}(\boldsymbol{x})=\overline{\boldsymbol{\varepsilon}} \cdot \boldsymbol{x}+\boldsymbol{u}^{*}(\boldsymbol{x}), \quad(\mathrm{KUBC}): \boldsymbol{u}(\boldsymbol{x})=\overline{\boldsymbol{\varepsilon}} \cdot \boldsymbol{x}
$$

In these equations, $\overline{\boldsymbol{\varepsilon}}$ denotes the average strain in the cubic cell, whereas $\boldsymbol{u}^{*}(\boldsymbol{x})$ represents an $L$-periodic displacement field that accounts for the field fluctuations and has volume average equal to zero. The PBC in Eq. (1) are automatically generated by 
NETGEN and are implemented using the "**quation" command in Abaqus as described by Danas in Ref. [51].

The homogenized fourth-order elastic stiffness tensor $\mathbb{C}$ is calculated from the average stress and strain fields using the constitutive equation [52]

$$
\langle\sigma(x)\rangle=\mathbb{C}\langle\varepsilon(\boldsymbol{x})\rangle,
$$

where \langle\rangle denotes the volume average of the stress $\boldsymbol{\sigma}(\boldsymbol{x})$ and strain field $\boldsymbol{\varepsilon}(\boldsymbol{x})$. To compute $\mathbb{C}$, we run six independent calculations [49], where a uniform macroscopic strain is applied along a specific direction, i.e. $\overline{\varepsilon_{i j}}$ with $i j=11,22,33,12,23,13$ (using the Voigt notation). The isotropized bulk and shear moduli, hereinafter denoted as $\kappa$ and $G$ respectively, are then computed by $\kappa=\mathbb{C}: \mathbb{J} / 3, G=\mathbb{C}: \mathbb{K} / 10$, with $\mathbb{I}, \mathbb{K}$ being the fourth-order hydrostatic and deviatoric projection tensors, respectively, of the fourth-order identity tensor $\mathbb{I}_{i j k l}=(1 / 2)\left(\delta_{i k} \delta_{j l}+\delta_{i l} \delta_{j k}\right)$ $(i, j=1,2,3)$. In addition, since our porous architectures are random, we quantify the deviation from isotropy of the numerical fourth-order elastic stiffness tensor $\mathbb{C}$, using the scalar parameter $\delta_{\text {iso }}=\left\|\mathbb{C}^{\text {iso }}-\mathbb{C}\right\|_{F} /\|\mathbb{C}\|_{F}$ (see Ref. [45]). In this last relation, $\|\mathbb{A}\|_{F}=\sqrt{\operatorname{Tr}\left(\mathbb{A} \cdot \mathbb{A}^{T}\right)}$ is the Frobenius norm of the tensor $\mathbb{A}$, and $\mathbb{C}^{\text {iso }}$ is the isotropic stiffness tensor obtained by projecting the actual stiffness tensor $\mathbb{C}$ along the $\mathbb{J}$, $\mathbb{K}$ spaces, i.e. $\mathbb{C}^{i s o}=3 \kappa \mathbb{J}+2 G \mathbb{K}$. In the current calculations reported in the present study, the numerical deviation from isotropy is found to be less than $\delta_{\text {iso }}<0.6 \%$, thus making the proposed microstructures isotropic.

Following those definitions, in a recent study [44], we proposed a roadmap for determining the size of the experimental RVE for random porous microstructures with monodisperse spheres up to $30 \%$ in volume. For these systems, the size of the RVE is univocally defined by a single geometrical parameter, namely the ratio $D / L$ between 
the void diameter $D$ and the characteristic length $L$ of the cubic cell [42], [49]-[50]. In Figure 3 of Ref. [44], we reported values of this ratio that lead to a converged RVE for every porosity explored. To compute these values, we progressively decreased the diameter of the voided spheres embedded in the cubic cell with fixed length $L=12$ $\mathrm{mm}$ and porosity $c$, until we obtained converged estimates for elastic moduli, i.e. estimates that are independent of the applied boundary conditions (PBC and KUBC).

The study of the converged RVE for polydisperse systems is however less straightforward, as these microstructures depend on multiple geometrical parameters. For the problem under consideration, these are the diameter $D_{i}$ and the number $N_{i}$ of the inclusions of the different pore families (i.e. different sizes). In this study, we determine the converged RVE for porous media with polydisperse voids/inclusions by modifying the protocol in [44] to account for polydispersity. Specifically, our strategy now consists in decreasing progressively the diameter $D_{\max }$ of the biggest pores in the RVE, with fixed length $L$ and porosity $c$, until convergence (up to a tolerance) of the elastic moduli is achieved by means of the two previously described boundary conditions PBC and KUBC. It is important to note at this point that a fixed value of the length of the cubic cell, i.e. $L=12 \mathrm{~mm}$, is imposed by the available axial extensometer (see Section 2.4), which has a nominal gage length of $10 \mathrm{~mm}$ with a travel of $\pm 2 \mathrm{~mm}$.

\subsection{Fabrication}

We fabricate the above-described 3D microstructures by $3 \mathrm{D}$ printing using the polymer printer EDEN 260VS from Stratasys (see Figure 1b-c). Our 3D-printer uses a PolyJet technology, where micrometric sized droplets (i.e. $\sim 16 \mu \mathrm{m}$ ) of liquid photopolymer are deposited layer by layer onto a build tray and cured instantly under UV light. The smallest sphere diameter that can be 3D-printed using this process is 
about $\sim 250 \mu \mathrm{m}$, see Figure 1c. Specifically, the employed matrix material is a glassy polymer with trade name VeroWhitePlus (Stratasys). Prior to testing, the matrix Young's modulus $\mathrm{E}_{\mathrm{m}}$ and Poisson's ratio $v_{\mathrm{m}}$ were measured via multi-step relaxation testing at room temperature (see Section 4 in Ref. [44]), and values were found to be respectively $\mathrm{E}_{\mathrm{m}}=1270 \pm 120 \mathrm{MPa}$ and $v_{\mathrm{m}}=0.42 \pm 0.02$.

Since 3D-printed parts are built layer by layer, a previous layer to build upon is required. To this end, the closed-cell pores embedded in the microstructures are printed using a support material that allows a 3D spherical inclusion to be fabricated (i.e. the nearly transparent phase in Figure 1c). The use of the support material to fabricate internal porous-like geometries is instrumental in current inkjet 3D printing. Similar to the excess powder or liquid in powder- and liquid-based additive manufacturing technologies, the support material occupies the closed cells. The gellike support material, with commercial name SUPP705 (Stratasys), has a tensile modulus $\mathrm{E}_{\mathrm{s}}=1.3 \pm 0.1 \mathrm{MPa}$ and Poisson's ratio $v=0.25$. The obtained modulus is almost thousand times smaller than that of the VeroWhitePlus matrix, which implies (after rudimentary analytical calculations such as the Hashin-Shtrikman or Voigt bounds), that the composite material behaves effectively as a porous material. We anticipate here that the difference that is found in calculating the Hashin-Shtrikman bounds between the porous matrix and the matrix with soft inclusions is very small, and notably below $0.1 \%$ (see Figure 3 for a more detailed discussion). These results are also corroborated in the following by the corresponding FE calculations.

Specifically, the moduli of the support material are measured experimentally using 5mm-thick strips printed out of this gel-like material. Every strip specimen is fabricated by $3 \mathrm{D}$-printing length-wise a U-shaped sandwich structure, whereby a 5 mm-thick empty core is enclosed between two $0.2 \mathrm{~mm}$-thick VeroWhitePlus layers. 
The strip is then easily removed manually, and mounted onto the tensile testing machine by means of two rigid heads printed out of VeroWhitePlus material. A somewhat alternative investigation of the effect of the support material on 3D-printed microstructures with pore features is also reported in Section 4 of Ref. [44] and corroborates independently the present findings.

\subsection{Tensile multi-step relaxation testing}

The elastic properties of the 3D-printed porous materials, namely the Young's Modulus and the Poisson's ratio, are measured by multi-step tensile relaxation experiments. The experiments are conducted in displacement control at a quasi-static strain rate $\overline{\dot{\varepsilon}}=10^{-5} \mathrm{~s}^{-1}$, and consist of seven relaxation steps at average strain increment of $0.1 \%$ for all volume fractions except $c=0.82$. In this last case, a displacement corresponding to $0.05 \%$ average axial strain is applied in order to prevent large straining of the thin matrix ligaments between neighboring pore-inclusions. To pilot the experiments, we use an in-built computer program. Specifically, at each step of relaxation the difference between two consecutive force measurements at 3 min-time intervals is computed, and the material is considered to be at its equilibrium state if such difference is smaller than $\sim 4 \mathrm{~N}$. To measure the force signal, we employ a 1.5 $\mathrm{kN}$ load transducer with accuracy $\pm 0.1 \mathrm{~N}$, which is mounted onto the fixed platen of an MTS servo-hydraulic uniaxial machine. The Young's modulus is then determined through linear regression of the ground elasticity points measured at each relaxation step, whereas the Poisson's ratio is computed as the slope of transverse-axial strain curves, see respectively Fig. $7 \mathrm{a}$ and $7 \mathrm{~b}$ in Ref. [44]. During the experiments, we measure simultaneously and independently the axial and radial strain signals respectively by means of a MTS 632.13F-20 (accuracy $\pm 0.0075 \mathrm{~mm}$ ) and an Epsilon 3475-025-M-ST (accuracy $\pm 0.1 \mathrm{~mm})$ transducer. 


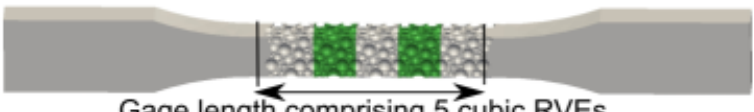

Gage length comprising 5 cubic RVEs

$\overline{12 \mathrm{~mm}}$

Figure 2: (Top) Virtual and (bottom) 3D-printed test specimens with gage length comprising five cubic RVEs. The RVEs have characteristic length $L=$ $12 \mathrm{~mm}$.

RVEs with characteristic length $L=12 \mathrm{~mm}$. The generated RVEs are assembled length-wise and are enclosed between the sample's heads, which, in turn, have a solid section in order to ease the specimen mounting on the uniaxial testing machine, see Figure 2. Prior to fabrication, the virtual test specimens are converted into stereolithography format for subsequent 3D printing.

\section{Results}

\subsection{FE results for the effective bulk and shear moduli}

Before we proceed to the experiments, we first carry out an extensive computational study to estimate the effective elastic moduli of composites containing random distributions of polydisperse spherical voids embedded in a linear elastic matrix. Using fairly significant computational resources, we explore numerically porosities ranging from $c=0$ to $c=0.82$. We emphasize here that no exact analytical solutions are available today (except for some special theoretical microstructures [53]) for the effective elastic moduli of general random porous solids, thus making the use of FE simulations instrumental.

The normalized effective shear $G / G_{m}$ and bulk $\kappa / \kappa_{m}$ moduli (where the subscript " $m$ " denotes the matrix phase) are shown as a function of the matrix volume fraction, 1 $c$, in Figure 3. The data points, depicted by solid circles, correspond to the average values of four different realizations and exhibit a very small standard deviation 
(below $0.1 \%$ ) as shown by the error bars. Moreover, for all porosities analyzed here the deviation from isotropy is less than $\delta_{i s o}<0.6 \%$ thereby indicating that our porous materials are fully isotropic solids. For comparison purposes, the theoretical HS bounds obtained for both an isotropic porous matrix (solid lines) and a matrix with soft inclusions with the measured elastic properties of the gel-like support material (dashed lines) are also reported in Figure 3. The difference between the FE predictions and the corresponding HS bounds remains small over the entire range of porosity explored. We also note in passing (see the inset in Figure 3) that the difference that is found by calculating the HS bounds for the porous matrix and the matrix with the soft inclusions is very small and notably in the same order as the contrast between the phases (i.e. $\approx 10^{-3}$ ).

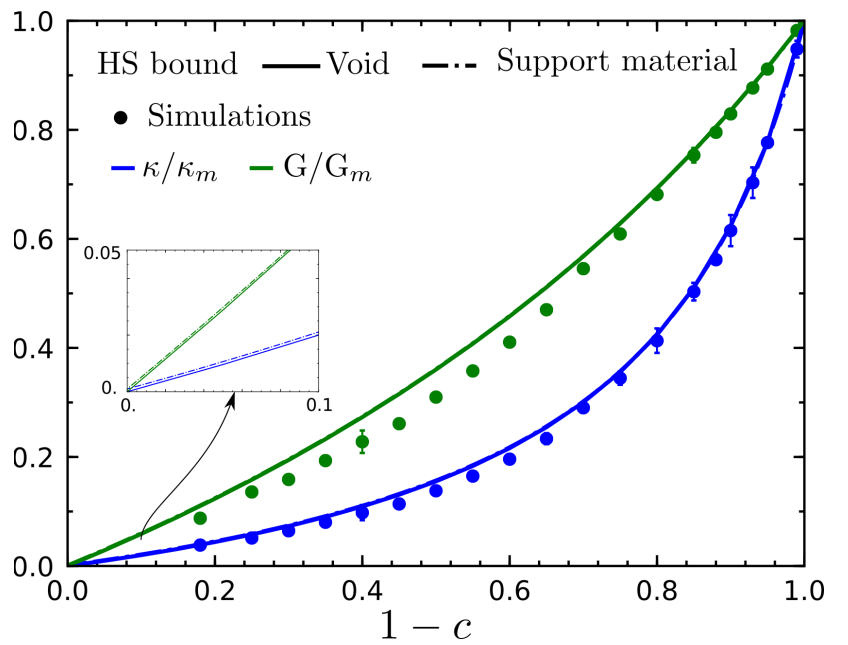

Figure 3: Plots of the normalized shear $\left(\mathrm{G} / \mathrm{G}_{\mathrm{m}}\right)$ and bulk modulus $\left(\kappa / \kappa_{\mathrm{m}}\right)$ vs. the matrix volume fraction $(1-c)$. The data points are obtained from $\mathrm{FE}$ periodic unit-cell simulations (PBC). The Hashin-Shtrikman upper bound for both an isotropic porous matrix (solid lines) and a matrix with soft inclusions (dashed lines) are also shown for each modulus using identical colors.
Moreover, this difference decreases gradually for moderate to low porosities $(0<c<0.4)$ and for large ones $(c>0.6)$. Rather interestingly, this difference is found to be maximum for intermediate values of the porosity, i.e, $0.4<\mathrm{c}<0.6$. We also note that the numerical bulk modulus is in much better agreement with the HS bounds than the shear modulus.

\subsection{Determination of the converged RVEs for experiments}

In Figure 4, we report the results of the RVE convergence study. Specifically, in Figure 4a-d, we discuss the process of obtaining a converged RVE for a 
representative porosity $c=0.6$. Following the method described in Section 2.2.1, we realize different cubic cells with decreasing diameter $D_{\max }$ (Figure 4a), whereas the effective elastic moduli are obtained from the FE simulations by applying KUBC and PBC (see Section 2.2.1). The computed bulk $\left(\kappa / \kappa_{\mathrm{m}}\right)$, shear $\left(\mathrm{G} / \mathrm{G}_{\mathrm{m}}\right)$ and Young's $\left(\mathrm{E} / \mathrm{E}_{\mathrm{m}}\right)$ moduli normalized by the corresponding matrix moduli are plotted as functions of $D_{\max }$ in Figure $4 \mathrm{~b}-\mathrm{d}$. In these figures, the diameter of the smallest voids $D_{\min }$ is used as secondary horizontal axis. For illustration purposes, we report in the inset of Figure $4 \mathrm{~b}$ the evolution of the ratio $D_{\max } / D_{\min }$ as a function of $D_{\max }$. To ensure statistical representativeness, each data point is the average of five simulations onto different realizations.

Collectively, the data in Figure $4 b-d$ exhibit similar trends with values of the KUBC estimates converging rapidly to the predictions of periodic unit-cell simulations (PBC) as $D_{\max }$ of the spherical voids decreases. Note that the decrease of the largest pore diameter $D_{\max }$ in the RSA algorithm leads to an increase of the total number of pores in the RVE (see Figure 4a), which leads to a rapidly increasing size of calculations. For every porosity analyzed in this study, we choose the value of $D_{\max }$ for which the difference in the KUBC and PBC moduli estimates is within a $2 \%$ deviation, as the largest pore size that leads to a representative cubic cell.

In Figure 4e, we summarize the results of the convergence study conducted for porosities in the range of $0 \leq c \leq 0.82$. Specifically, we show the evolution of both $D_{\max } / D_{\min }$ and $D_{\min }$ (blue and red curves respectively) as a function of the matrix volume fraction, i.e. $1-c$. At small porosities, i.e. $0 \leq c \leq 0.35$, and in agreement with our earlier study on monodisperse voided RVEs [44], the ratio $D_{\max } / D_{\min }$ remains constant and equal to $D_{\max } / D_{\min }=1$ (with $D_{\min }=1.24 \mathrm{~mm}$ ), thereby revealing that a single size of voids (i.e. a monodisperse distribution) is sufficient to deliver 
converged RVEs. This finding is also in agreement with earlier studies [43],[49],

where the effective elastic properties for monodisperse and polydisperse spherical inclusions at equal volume fraction were found to be nearly identical. On the other hand, for $\mathrm{c} \geq 0.4$, polydispersity is essential to achieve high values of porosity.

a)

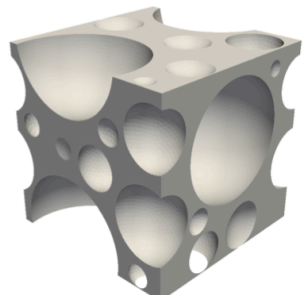

b)
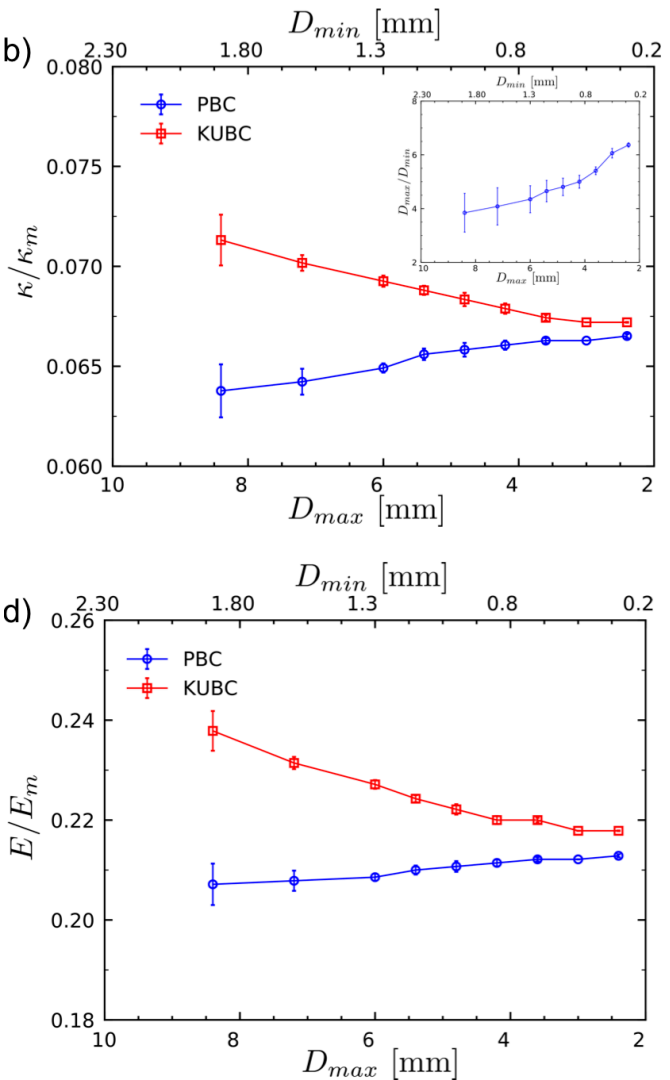
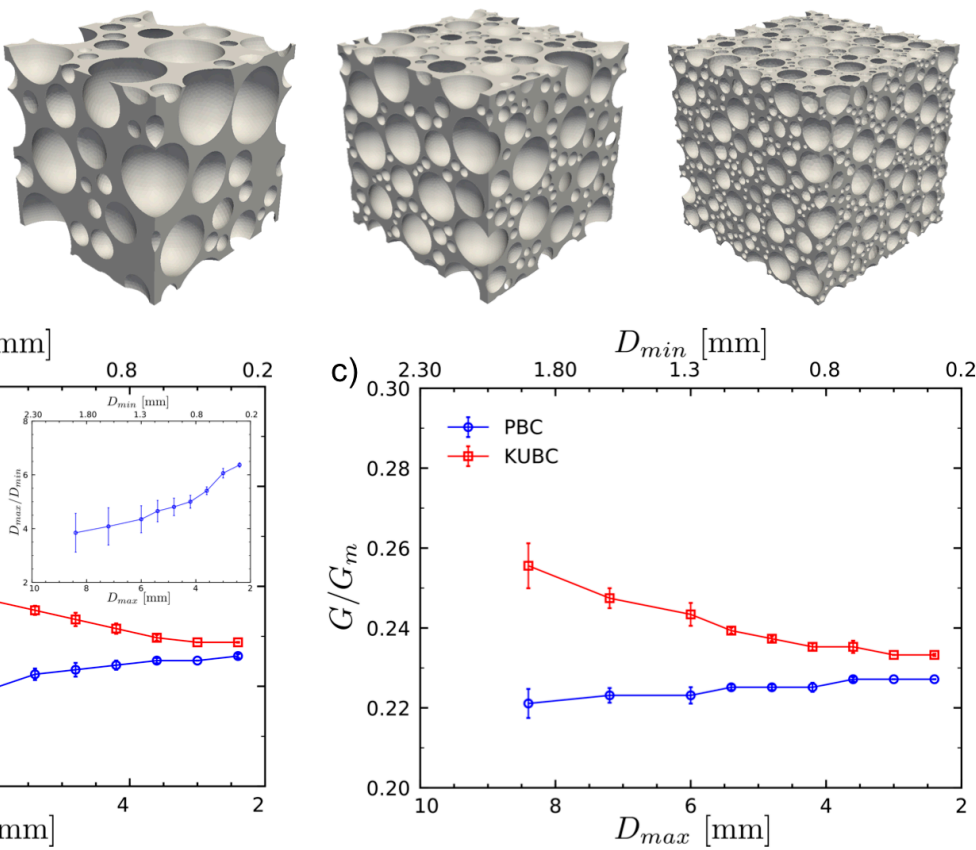

e)

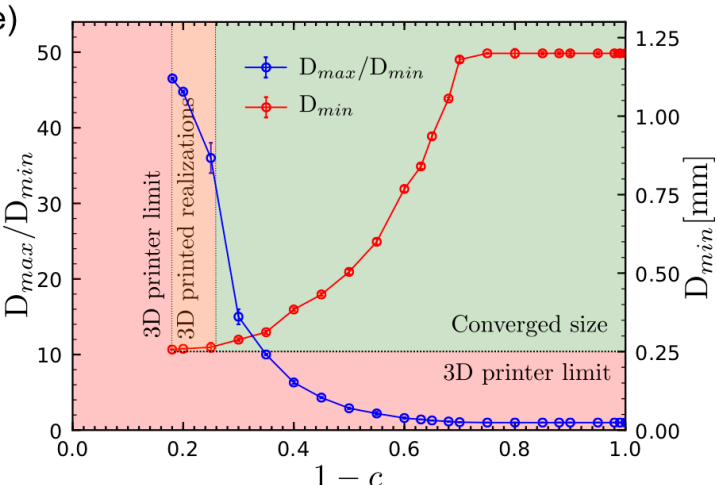

Figure 4: (a) Realizations of microstructures containing a random distribution of polydisperse spherical voids at porosity $\mathrm{c}=0.6$. Geometrical parameters for these microstructures (from left to right) are: (i) $\mathrm{D}_{\max }=8.4 \mathrm{~mm}$ and $\mathrm{D}_{\max } / \mathrm{D}_{\min }=4$ (ii) $\mathrm{D}_{\max }=6.0 \mathrm{~mm}$ and $\mathrm{D}_{\max } / \mathrm{D}_{\min }=4.5$, (iii) $\mathrm{D}_{\max }=4.8 \mathrm{~mm}$ and $D_{\max } / D_{\min }=5$, (iv) $D_{\max }=2.3 \mathrm{~mm}$ and $\mathrm{D}_{\max } / \mathrm{D}_{\min }=6.5$. (b-d) Results for the (b) bulk, (c) shear and (d) axial normalized moduli obtained from FE simulations with periodic (PBC) and kinematically uniform (KUBC) boundary conditions. Data points are the average of five realizations. The inset in figure (b) shows the evolution of the ratio between the diameters of the biggest and smallest pores. (e) Results of the convergence study for a large range of porosity, $0 \leq \mathrm{c} \leq 0.82$ showing the ratio $D_{\max } / \mathrm{D}_{\min }$ and $\mathrm{D}_{\min }$ as a function of the porosity. Recall that the smallest $3 \mathrm{D}$-printable diameter is $\mathrm{D}_{\lim }=0.25 \mathrm{~mm}$. 
In fact, Figure $4 \mathrm{e}$ shows a rapid decrease of the converged $D_{\min }$ accompanied by a simultaneous increase of the ratio $D_{\max } / D_{\min }$. This last result indicates that, at large porosities, the material under study is inherently multiscale and with a very large number of different sizes. Moreover, values of $D_{\max }$ within the range of porosities explored can be readily inferred from Figure 4e. Specifically our study shows that, the size ratio of the largest to smallest inclusion, $D_{\max } / D_{\min }$, increases rapidly with increasing porosity when $\mathrm{c}$ becomes larger than $60 \%$ (or $1-c<0.4$ ). In turn, our analysis reveals that at very large porosities (i.e. $c>0.75$ ) the ratio of the minimal inter-void ligament $t$ (with $t=150 \mu \mathrm{m}$ ) to $D_{\max }$ is very small, namely $t / D_{\max }<1 / 60$. Finally, we clarify here that for $c>0.75$ the convergence of the RVE is mainly statistical and is achieved by analysing a large number of different realizations. The reason is that at $c=0.82$, we reach the smallest sphere diameter, $D_{\min }=D_{\text {lim }}=250 \mu \mathrm{m}$, which is attainable by our 3D printer (see highlighted orange region in Figure 4e).

\subsection{Experimental results for the effective Young's modulus and Poisson's ratio}

In Figure 5a and Figure 5b, we show the experimental Young's modulus $E$ and Poisson's ratio $v$ of porous materials with matrix volume fraction $0.18 \leq 1-c \leq 1$ (or 0 $\leq c \leq 0.82$ ). The results are obtained as the average of at least four specimens and are reported together with their error bar. The latter corresponds to the standard deviation on the data. For comparison, the HS upper bounds for an isotropic porous solid (solid lines) alongside the FE estimates (solid symbols) are also reported. The latter are computed from data in Fig. 3 using the standard linear elastic isotropic Hooke's law, i.e., $E=9 \kappa G /(3 \kappa+G)$ and $v=(3 \kappa-2 G) /(2(3 \kappa-2 G))$.

The experimental data are in very good quantitative agreement with the numerical FE predictions and lie very close to the HS bounds. Notably, the maximum deviation of the experimental Young's modulus $E$ from the theoretical HS bound is observed at 
moderate porosity ( $c=0.5$ ), where the data exhibit the highest variability (see the error bar). On the other hand, the measured values of the Poisson's ratio exhibit an increasing deviation from the average as the volume fraction of voided inclusions increases (see Figure 5b). The observed sensitivity for the measurements of the transverse strain at large volume fraction of inclusions is consistent with an earlier study on highly loaded particulate composites [54]. Moreover, data in Figure 5b

a)

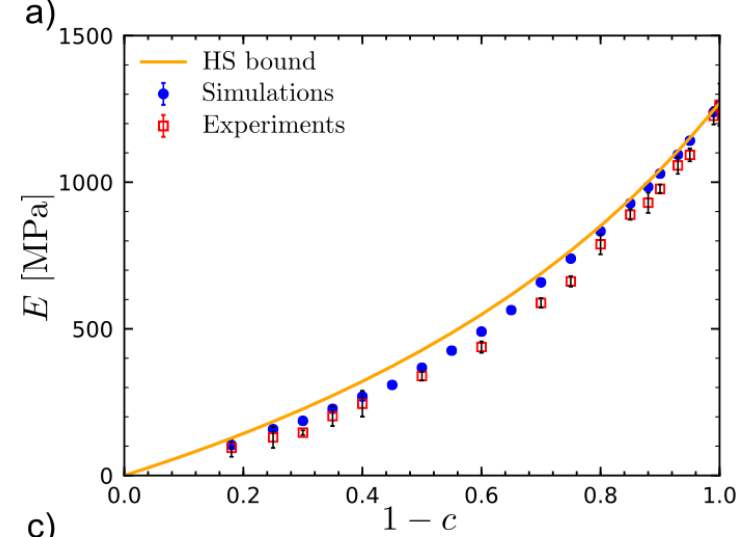

c)
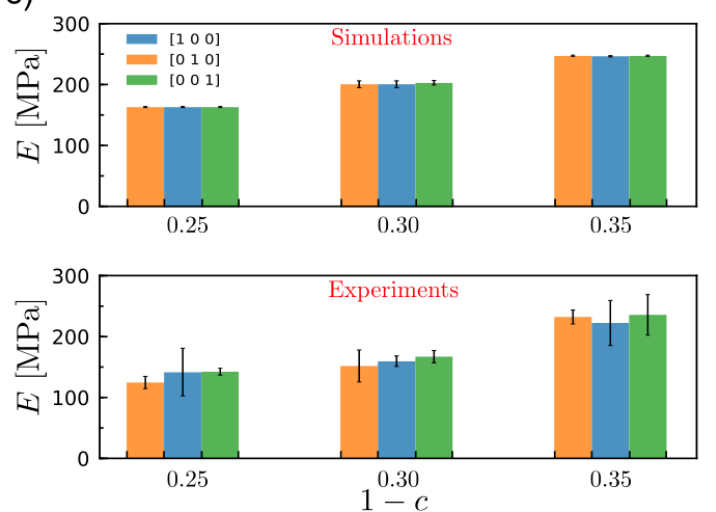

b)

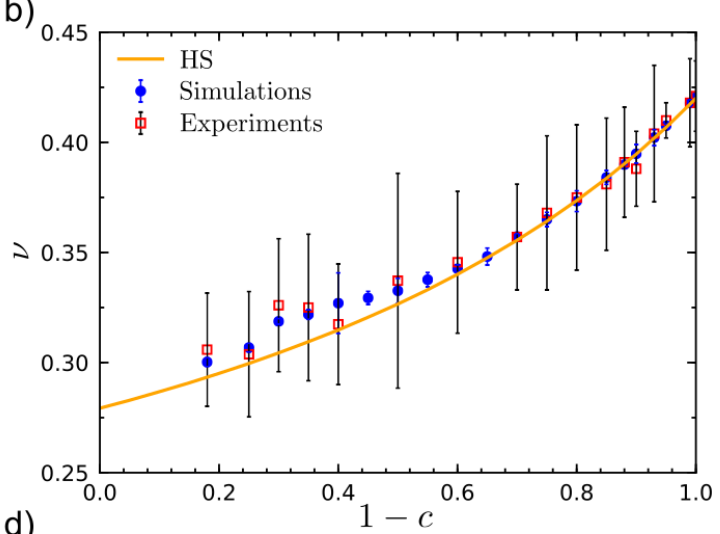

d)

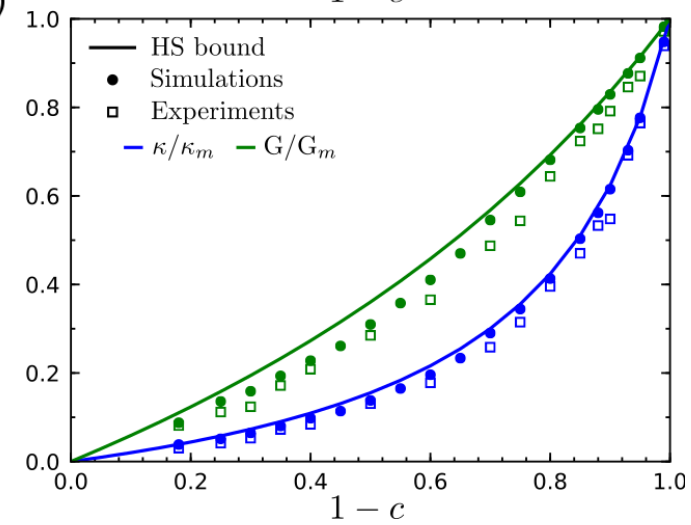

Figure 5: Experimental (a) Young's modulus and (b) Poisson's ratio as a function of 1-c. Results of the FE simulations (solid symbols) together with the Hashin-Shtrikman bounds (solid lines) are also included for comparison. (c) Numerical estimates (top) and experimental measurements (bottom) of the Young's moduli along three different directions for moderate-to-high porosity volume fractions. (d) Normalized bulk and shear moduli calculated using the average values of the experimental Young's modulus and Poisson ratio values in (a) and (b) respectively.

display a small departure from the HS curve (here not a bound) for $c \geq 0.4$.

An important aspect of the experimental study (similar to the numerical one) is the isotropy of the 3D-printed RVEs. It is noted here that this is a non-trivial analysis since the RVEs under study do not exhibit any symmetry planes, as is the case in highly periodic trusses and lattices. Moreover, from the corresponding numerical 
study, we observe that if such RVEs exhibit similar moduli along the three cubic directions they also have similar values for the effective simple shear moduli as a consequence of the random dispersion of the inclusions. Therefore, in order to probe the degree of isotropy in our 3D-printed composites, we measure the Young's modulus along the three orthogonal axes of the cubic RVE at selected volume fractions of void-like inclusions, e.g., $c=0.65,0.7,0.75$, where the RVEs comprise a very large number of void sizes. This is achieved by first rotating the generated cubic cell along its three axes and then by using each rotated cell to construct the virtual test sample. The latter is then 3D-printed and tested. Attention is here limited to the axial stiffness, since measurements of the Poisson's ratio along different directions are likely to be clouded by the larger scatter of data (see Figure 5b). For comparison, we repeat exactly the same process with the numerical RVEs. Results are reported in Figure $5 \mathrm{c}$ and are averages of three samples. As observed, the measured experimental degree of anisotropy is small. Specifically, the difference between the largest and smallest average experimental moduli is equal to 12,9 and $6 \%$ for $c=0.75,0.7$ and 0.65 , respectively. These deviations are in the order of the experimental scatter of data for the pure matrix response (see Section 4 in Ref. [44]). On the other hand, differences in the numerical predictions are negligible. This is consistent with the results in Section 3.1, where a deviation of isotropy below $0.6 \%$ is reported.

\section{Discussion}

Random distributions of finitely polydisperse spherical voids (gel-like inclusions) embedded into a homogeneous elastic matrix produce microstructures with elastic moduli that lie close to the corresponding HS bounds within the range of porosity explored (see Figure 3 and Figure 5a). Moreover, these microstructures are isotropic 
as revealed by both experiments and simulations (see Figure 5c and Section 3.1). As for most of open-cells foams produced by replication process [13],[15], isotropy in our materials is a direct consequence of the microstructural randomness. This feature is in contrast with the periodicity of most of lattices and trusses composed of webs or trusses [9],[25]. To guide the discussion that follows, in Figure 5d, we compare the experimental and numerical normalized bulk $\left(\kappa / \kappa_{\mathrm{m}}\right)$ and shear $\left(\mathrm{G} / \mathrm{G}_{\mathrm{m}}\right)$ moduli for a large range of porosity, i.e., $0 \leq c \leq 0.82$. Specifically, we evaluate the experimental bulk and shear moduli via the linear elastic Hooke's law (i.e. $\kappa=E /(3(1-2 v))$ and $G=$ $E /(2(1+v)))$ using the average value of $E$ and $v$ in Figure 5a,b (i.e. data points without the error bar). Figure 5d clearly shows that the microstructures realized in this study are near-optimal under hydrostatic loading but are not maximally stiff under shear loading. Yet, the difference between the data points for $\mathrm{G} / \mathrm{G}_{\mathrm{m}}$ and the theoretical HS bound remains small over the entire range of porosities explored, and decreases rapidly for small and large $c$. Moreover, the strong agreement between FE and experiments for all porosities analyzed suggests that the FE analysis accurately captures the underlying local deformation mechanisms.

In turn, the small differences between the FE and the experiments can be attributed to two different distinct features. The first is directly linked to the experimental uncertainty in the elastic moduli of the base polymer matrix as these differences are in the same order. The second feature is related to the local nonlinear strains that are developed in the experimental material, whereas the FE study is carried out only in linear elasticity. Instead, it is highly unlikely that those small differences are a consequence of other factors such as the non-uniformity of the cell walls as observed for most of truss-lattices [25][27]), and internal damage (as reported for metallic open-cell foams [13], [55] at strains larger than 0.02). In fact, the present study, in 
agreement with prior investigations [44], shows that the size and shape of the spherical void-like inclusions are 3D-printed with very high accuracy (Figure 1). Moreover, the elastic constants in Figure 5 are measured at very low strains (well

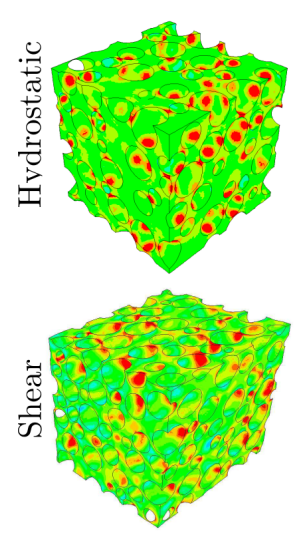

$c=0.4$

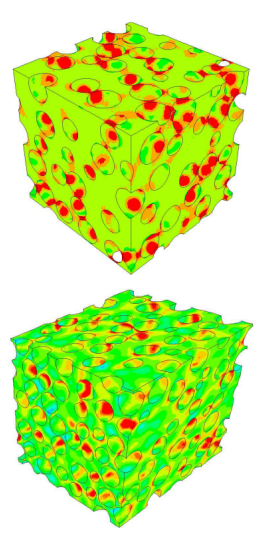

$c=0.5$

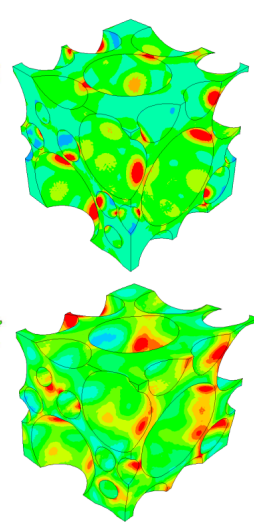

$c=0.65$

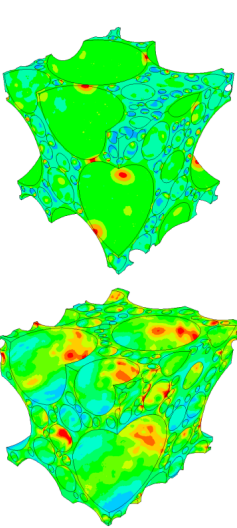

$c=0.75$

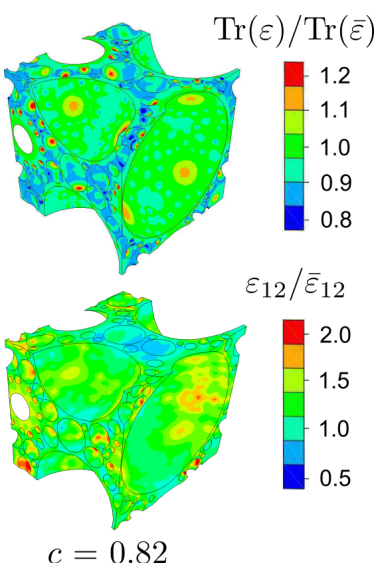

$c=0.82$

Figure 6: Strain contours of selected porous microstructures under hydrostatic and shear loadings. Here the bar notation is used to denote the macroscopic applied average strain $\bar{\varepsilon}$ in the RVE.

below 0.01 , see Section 2.4) to prevent damage of the thin matrix ligaments.

In order to rationalize further the underlying mechanism for the observed deviation of data from the theoretical predictions (Figure 5d), we use the results of FE simulations. Specifically, in Figure 6, we show local strain fields for selected porosities, $c=0.4,0.5,0.65,0.75$ and 0.82 , under macroscopic hydrostatic (upper row) and shear (lower row) PBC loading. We study, in particular, the deviation of the hydrostatic and shear local strains from their average counterparts. As observed in this figure, the strain deviations are much higher at moderate porosities, e.g., $c=0.4,0.5$, whereas the fields near the internal boundaries of the voids are found to become more uniform with increasing porosity (i.e., $c>0.6$ ) (or decreasing porosity, $\mathrm{c}<0.3$, not shown here but see [44]). Furthermore, the highest strain concentration occurs in the regions between voids reaching, more often than not, values that exceed significantly (more than two times) the applied macroscopic strain in agreement with numerous previous studies on porous microstructures [44] and foams [56]-[57]. Those local 
strain fluctuations in random closed-cell porous solids are a direct consequence of the interaction between the closely packed spherical voids [44], [56]. At very high porosity however, i.e. at $c>0.65$, the matrix ligament between the voids becomes very thin leading to gradually more uniform strain fields. Similar observations have also been made in the context of muscle geometries [58],[59] and close-cell random foams produced by Voronoi tessellation [60]. For these microstructures, Roberts and Garboczi [60] have shown, using FE simulations, that a simple scaling relation of the elastic moduli with relative density lacks clear physical significance.

To correlate the observed local strain fields with the porous microstructure, we report in Figure 7 the simulated microstructures at porosity $\mathrm{c}=0.2,0.5$ and 0.82 together with the numerical strain contours for hydrostatic loading. In agreement with

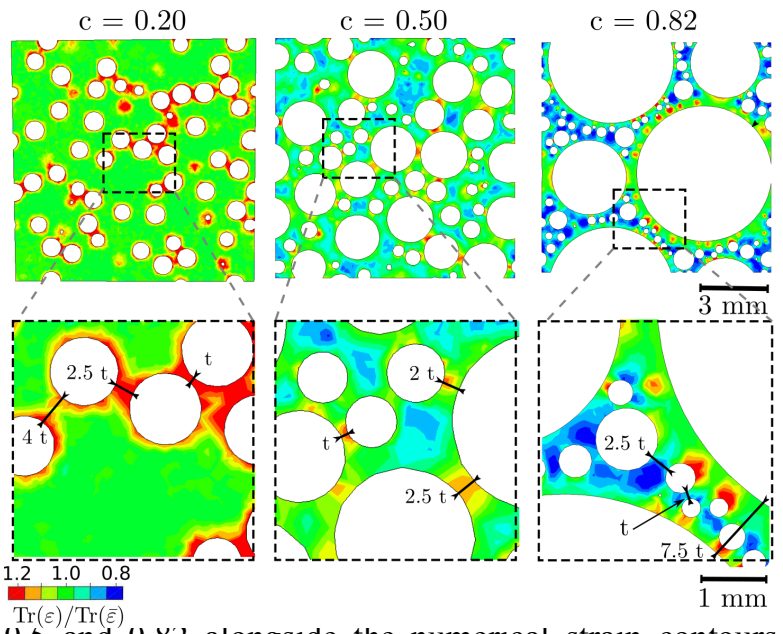

0.5 and 0.82 alongside the numerical strain contours under hydrostatic loading. For the definition of the bar notation see Figure 6. the analysis in Figure 4e, with increasing porosity the microstructure evolves from a random monodisperse to a finitely polydisperse distribution of spheres. As discussed earlier, the largest void diameter is observed to increase as the porosity increases from $\mathrm{c}=0.2$ to $\mathrm{c}=0.82$ (Figure 7). Collectively, the numerical micrographs in Figure 7 reveal microstructural features similar to those reported for other random closed-cell foams produced by conventional foaming process. Prior studies on foamed polymers $[56],[61]-[62]$, glass [63] and ceramics [64] with porosity between 0.3 and 0.75 , show that the microstructure of these materials consists of a homogeneous (random) distribution of nearly spherical bubbles. These bubbles are found to be uniform both 
in size and shape at low porosity [56],[61], but gradually become polydisperse as the porosity increases [61]-[62]. Interestingly, similar to random closed-cell foams $[56],[61]-[64]$, the present porous composites (see the magnifications in the bottom row of Figure 7) clearly show that there is no unique minimal thickness for the interinclusion ligament $t$. This is a direct consequence of the void polydispersity and randomness. At high porosity, e.g. $\mathrm{c}=0.82$ (Figure 7), the matrix ligament between two neighbouring large voids contains a number of smaller inclusions as is the case also for foamed polymers and ceramics at low density in Refs. [61]-[62],[64].

Finally, in Figure 8 we compare, at equal matrix volume fraction $1-c$, the measured normalized axial stiffness of the present closed-cell random porous materials with recent porous materials as well as with fully-stochastic foams produced by more conventional manufacturing processes (e.g. foaming and replication). As seen, the 3D-printed porous solids of this work exhibit normalized Young's modulus values that rival those of closed-cell stochastic foams. The study on the porous glass [63] (whose moduli are reported in Ref. [60] and in Figure 8) alongside experimental investigations on other foamed polymers [56],[61]-[62] indicate that the microstructure of these materials comprises similar geometrical features to those of the present composites. More interestingly, our materials are almost twice stiffer than most open-pore microcellular foams [15] and two to five times stiffer than two of the most performing metamaterials demonstrated today. The latter are the nano- and macroscale octet-truss lattices fabricated by 3D laser writing [65]. Hence, the present random 3D-printed porous materials are promising candidates in terms of relative stiffness per unit-volume. 


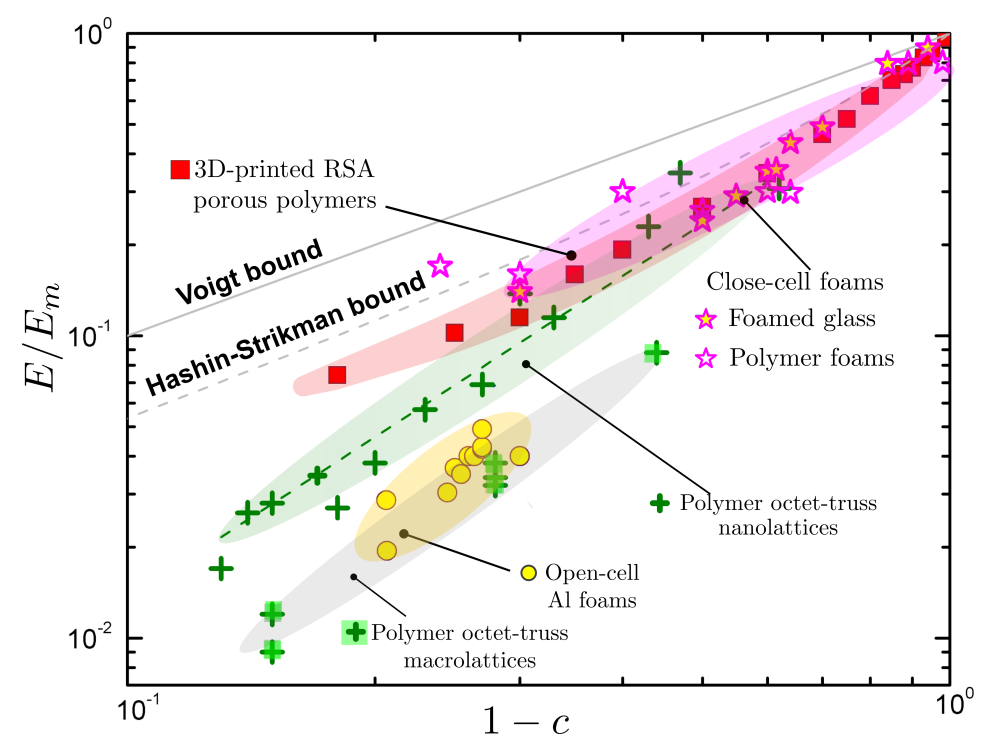

Figure 8: Property space map of the Young's modulus versus matrix volume fraction, $1-c$, comparing the present isotropic 3D-printed porous polymers (red solid squares) with other closed-cell and opencell foams as well as with nano- and macro lattices of similar porosity.

The advantages they

offer over other fully stochastic cellular solids, i.e. foams, produced by conventional processing routes (for which data in Figure 8 are taken from [60]) are many. These notably are the precision of the size and shape of the pore-inclusions as well as the ability to reach very large and precise volume fractions in a straightforward and controlled manner.

Moreover, by virtue of their fully random architecture the proposed materials offer the added benefit of being fully isotropic, whereby most of today's lightest and stiffest metamaterials of the same porosity are not [10], [24]-[29],[65]. On the other hand, the present materials are not cellular in the sense of relative density (but behave as such in the mechanical sense). As explained before, the reason is that the inclusions are made of a gel-like material that, despite having low axial stiffness and behaving mechanically as a porous-like phase as shown in section Section 3.3, it has almost the same density of the matrix material. In order to overcome this issue, several ideas are currently being explored such as introducing a minor connectivity between the inclusions and using a chemically soluble support material. Nonetheless, this is an effort at the very early stages and is left to a future study. 


\section{Conclusions}

In this study, we probe the elastic deformation of 3D-printed random porous architectures consisting of non-overlapping, finite polydisperse spherical void-like inclusions embedded in a polymer matrix. The void-like spherical inclusions are built by the 3D-printer using a gel-like support material, whose measured elastic moduli are in high contrast (1:1000) with those of the glassy polymer matrix. Specifically, we explore volume fractions of porosity that extend from $c=0$ to 0.82 . The microstructures are generated using a modified random sequential adsorption algorithm, where the spherical voided inlusions, with a gradually decreasing size, are added sequentially and randomly in a cubic cell. Using 3D-printed dog-bone specimens comprising five converged representative volume elements (RVE), we measure the elastic Young's modulus and the Poisson's ratio during tensile multi-step relaxation testing at room temperature.

The major finding of this study is that our microstructures provide effective elastic moduli that lie very close to the theoretical Hashin-Shtrikman upper bound for all range of porosities considered. Moreover, the proposed composites are isotropic. Experiments (for selected values of porosity) reveal that the differences in the axial stiffness measured along the three main axes of the cubic cell are higher for intermediate porosities, for instance in the order of $10 \%$ for $c=0.65$. Since our results show a very good quantitative agreement between experiments and simulations, we use the results of $\mathrm{FE}$ to rationalize the observed deviation of the moduli from the theoretical predictions. Notably, we analyze the local strain field for selected porous microstructures under macroscopic hydrostatic and shear loadings. Our analysis confirms that strain localization is higher at moderate porosity, whereas the fields become progressively more uniform as porosity increases. 
Finally, a detailed comparison of the normalized Young's modulus of our porous microstructures with available data in the literature highlights their structural advantage. Due to the fact that our microstructures are generated using a highly versatile random generation algorithm, the size, shape and orientation of the voidedinclusions can be precisely controlled during the material design. This capability engenders a virtually unbounded potential for the design of novel (meta)materials, where architecture can be efficiently optimized to achieve a target objective at minimal weight. Indeed, the diversity of microstructures that can be produced by this route is high. This attribute, coupled with the wide latitude in selection of the material from which the porous architecture can be printed (including metals, glass and ceramics), makes our approach ideally suited to explore a wealth of microstructures that cannot be synthetically reproduced in any other way, from rocks and biomaterials to even foamed food.

\section{Acknowledgments}

The authors acknowledge support of the European Research Council (ERC) under the European Unions Horizon 2020 research and innovation program (grant agreement no. 636903). O.Z. and K.D. also acknowledge support from TOTAL S.A. The computational part of this work was also supported by the ANR under contract number ANR-10-EQPX-37.

\section{References}

[1] Schurig, D., Mock, J. J., Justice, B. J., Cummer, S. A., Pendry, J. B., Starr, A. F., Smith, D. R. Metamaterial electromagnetic cloak at microwave frequencies. Science (2006), 314(5801), 977-980.

[2] Landy, N. I., Sajuyigbe, S., Mock, J. J., Smith, D. R., Padilla, W. J. Perfect metamaterial absorber. Phys. Rev. Lett (2008), 100, 207402.

[3] Smith, D. R., Pendry, J. B., Wiltshire, M. C. Metamaterials and negative refractive index. Science (2004), 305(5685), 788-792.

[4] Chen, H., Chan, C. T. Acoustic cloaking in three dimensions using acoustic metamaterials. Applied physics letters (2007), 91(18), 183518. 
[5] Lee, J. H., Singer, J. P., Thomas, E. L. Micro/nanostructured mechanical metamaterials Adv. Mater. (2012), 24, 4782-4810.

[6] A.A. Zadpoor. Mechanical meta-materials. Mater. Horiz. (2016), 3 (5), 371-381.

[7] Kolken, H. M., Janbaz, S., Leeflang, S. M., Lietaert, K., Weinans, H. H., Zadpoor, A. A. Rationally designed meta-implants: A combination of auxetic and conventional meta-biomaterials. Mater. Horiz. (2018), 5(1), 28-35.

[8] Ahmadi, S. M., Hedayati, R., Li, Y., Lietaert, K., Tümer, N., Fatemi, A., Zadpoor, A. A. Fatigue performance of additively manufactured meta-biomaterials: The effects of topology and material type. Acta biomaterialia (2018), 65, 292-304.

[9] Schaedler, T. A., Carter, W. B. Ultralight metallic microlattices. Annu Rev Mater Res. (2016), 46, 187-210.

[10] Compton, B. G., Lewis, J. A. 3D-printing of lightweight cellular composites. Adv Mater. (2014), 26(34), 5930-5935.

[11] Wang, L., Lau, J., Thomas, E. L., Boyce, M. C. Co continuous composite materials for stiffness, strength, and energy dissipation Adv Mater. (2011), 23(13), 1524-1529.

[12] Gibson, L. J., Ashby, M. F. Cellular solids: structure and properties. Cambridge Univ Press, (1999).

[13] San Marchi, C., Despois, J. F., Mortensen, A. Uniaxial deformation of open-cell aluminum foam: the role of internal damage. Acta Mat. (2004), 52(10), 2895-2902.

[14] San Marchi, C., Mortensen, A. Deformation of open-cell aluminum foam. Acta Mat, (2001), 49(19), 39593969.

[15] Despois, J. F., Conde, Y., San Marchi, C., Mortensen, A. Tensile behaviour of replicated aluminium foams. Adv Eng Mater, (2004), 6(6), 444-447.

[16] Diologent, F., Goodall, R., Mortensen, A. Creep of aluminium-magnesium open cell foam. Acta Mat, (2009). 57(3), 830-837.

[17] Combaz, E., Bacciarini, C., Charvet, R., Dufour, W., Dauphin, F., Mortensen, A. Yield surface of polyurethane and aluminium replicated foam. Acta Mat, (2010), 58(15), 5168-5183.

[18] Combaz, E., Mortensen, A. Fracture toughness of Al replicated foam. Acta Mat, (2010), 58(14), 45904603.

[19] Despois, J. F., Mortensen, A. Permeability of open-pore microcellular materials. Acta Mat, (2005), 53(5), 1381-1388.

[20] Conde, Y., Despois, J. F., Goodall, R., Marmottant, A., Salvo, L., San Marchi, C., Mortensen, A. Replication processing of highly porous materials. Adv Eng Mater, (2006), 8(9), 795-803.

[21] Mortensen, A., Fitzgerald, T. J. Method for producing a microcellular foam. U.S. Patent No 5,571,848, 1996.

[22] Deshpande, V. S., Ashby, M. F., Fleck, N. A. Foam topology: bending versus stretching dominated architectures. Acta Mat, (2001), 49(6), 1035-1040.

[23] Deshpande, V. S., Fleck, N. A., Ashby, M. F. Effective properties of the octet-truss lattice material. $J$ Mech Phys Solids, (2001), 49(8), 1747-1769.

[24] Bauer, J., Hengsbach, S., Tesari, I., Schwaiger, R., Kraft, O. High-strength cellular ceramic composites with 3D microarchitecture. Proc Nat Acad Sci, 2014, 111(7), 2453-2458.

[25] Meza, L. R., Das, S., Greer, J. R. Strong, lightweight, and recoverable three-dimensional ceramic nanolattices. Science, 2014, 345(6202), 1322-1326.

[26] Zheng, X., Lee, H., Weisgraber, T. H., Shusteff, M., DeOtte, J., Duoss, E. B., Kucheyev, S. O. Ultralight, ultrastiff mechanical metamaterials. Science, 2014, 344(6190), 1373-1377.

[27] Jang, D., Meza, L. R., Greer, F., Greer, J. R. Fabrication and deformation of three-dimensional hollow ceramic nanostructures. Nature Mater, 2013, 12(10), 893.

[28] Schaedler, T. A., Jacobsen, A. J., Torrents, A., Sorensen, A. E., Lian, J., Greer, J. R., Carter, W. B. Ultralight metallic microlattices. Science, 2011, 334(6058), 962-965.

[29] Han, S. C., Lee, J. W., Kang, K. A new type of low density material: Shellular. Adv Mater, 2015, 27(37), 5506-5511.

[30] Cherkaev, A. Variational Methods for Structural Optimization, Springer-Verlag, New York, 2000.

[31] Milton, G. W. The Theory of Composites, Cambridge Univ. Press, Cambridge, 2002.

[32] Hashin Z, Shtrikman A variational approach to the theory of the elastic behaviour of multiphase materials.S. J Mech Phys Solid, 1963, 127-140.

[33] Willis J.R., Bounds and self-consistent estimates for the overall properties of anisotropic composites.J Mech Phys Solids, 1977, 25, 185-202.

[34] Hashin Z., Analysis of composite materials-a survey. J Appl Mech., 1962, 481-505.

[35] Francfort G.A., Murat F., Homogenization and optimal bounds in linear elasticity. Arch Ration Mech Anal, 1986, 94:307-34.

[36] Norris, A. N., A differential scheme for the effective moduli of composites. Mech. Mater. (1985), 4, 1-16.

[37] Milton, G. W. in Homogenization and Effective Moduli of Materials and Media (eds Ericksen, J. L. et al.) 1986, 150-174 (Springer-Verlag, New York).

[38] Berger, J. B., Wadley, H. N. G., McMeeking, R. M. Mechanical metamaterials at the theoretical limit of isotropic elastic stiffness.Nature, 2017, 543(7646), 533.

[39] Tancogne-Dejean, T., Diamantopoulou, M., Gorji, M. B., Bonatti, C., Mohr, D. 3D Plate Lattices: An Emerging Class of Low Density Metamaterial Exhibiting Optimal Isotropic Stiffness. Adv Mater, 2018, 1803334 . 
[40] Talbot, J., Schaaf, P., Tarjus, G. Random sequential addition of hard spheres. Molecular Physics, 1991, 72(6), 1397-1406.

[41] Rintoul M, Torquato S. Reconstruction of the structure of dispersions. J Colloid Interface Sci., 1997,186:467-76.

[42] Segurado J, Llorca J. A numerical approximation to the elastic properties of sphere-reinforced composites. J Mech Phys Solids, (2002), 50.

[43] Lopez-Pamies O, Goudarzi T, Danas K. The nonlinear elastic response of suspensions of rigid inclusions in rubber: II-a simple explicit approximation for finite-concentration suspensions. J. Mech. Phys. Solids, (2013); 61:19-37.

[44] Zerhouni, O., Tarantino, M. G., Danas, K. Numerically-aided 3D printed random isotropic porous materials approaching the Hashin-Shtrikman bounds. Compos Part B-Eng, 2019, 156, 344-354.

[45] Anoukou, K., Brenner, R., Hong, F., Pellerin, M., Danas K. Random distribution of polydisperse ellipsoidal inclusions and homogenization estimates for porous elastic materials. Computers Struct, 2018, 210,87-101.

[46] Schöberl, J. NETGEN. An advancing front 2D/3D-mesh generator based on abstract rules. Computing and visualization in science, (1997). 1(1), 41-52.

[47] Suquet P., Elements of Homogenization for Inelastic Solid Mechanics. Cours au Centre International des Sciences Mécaniques. Udine. 1985. In E. Sanchez-Palencia, A. Zaoui (eds), Homogenization Techniques for Composite Media. Lecture Notes in Physics N0272. Springer-Verlag. Berlin. 1987. p.193-278.

[48] Mbiakop A., Constantinescu A., Danas K. (2015). A model for porous single crystals with ellipsoidal voids, J. Mech. Phys. Solids, 84, 436-467.

[49] Kanit, T., Forest, S., Galliet, I., Mounoury, V., Jeulin, D. Determination of the size of the representative volume element for random composites: statistical and numerical approach. Int J Solids Struct (2003). 40(13-14), 3647-3679.

[50] Kari, S., Berger, H., Rodriguez-Ramos, R., Gabbert, U. Computational evaluation of effective material properties of composites reinforced by randomly distributed spherical particles. Composite structures, (2007). 77(2), 223-231.

[51] Danas, K. Effective response of classical, auxetic and chiral magnetoelastic materials by use of a new variational principle. J Mech Phys Solids, 2017, 105, 25-53.

[52] Hill, R. Elastic properties of reinforced solids: some theoretical principles. J Mech Phys Solids, (1963). 11(5), 357-372.

[53] Shrimali, B., Lefèvre, V., Lopez-Pamies, O., A simple explicit homogenization solution for the macroscopic elastic response of isotropic porous elastomers, J Mech Phys Solids, (2019), 122, 364-380.

[54] Tarantino, M. G., Weber, L., Mortensen, A. Effect of hydrostatic pressure on flow and deformation in highly reinforced particulate composites. Acta Mater, 2016, 117, 345-355.

[55] Dillard, T., N'guyen, F., Maire, E., Salvo, L., Forest, S., Bienvenu, Y., Cloetens, P. 3D quantitative image analysis of open-cell nickel foams under tension and compression loading using X-ray microtomography. Philosophical Magazine, 2005, 85(19), 2147-2175.

[56] Youssef, S., Maire, E., Gaertner, R. Finite element modelling of the actual structure of cellular materials determined by X-ray tomography. Acta Mater, 2005, 53(3), 719-730.

[57] Caty, O., Maire, E., Youssef, S., Bouchet, R. Modeling the properties of closed-cell cellular materials from tomography images using finite shell elements. Acta Mater, 2008, 56(19), 5524-5534.

[58] Spyrou, L.A., Agoras M., Danas, K., J. A homogenization model of the Voigt type for skeletal muscle. Theor. Biology, 2017, 414, 50-61.

[59] Spyrou, L.A., Brisard, S., Danas, K. Multiscale modeling of skeletal muscle tissues based on analytical and numerical homogenization. J. Mech. Behav. Biomed. Mater., 2019, 92, 97-117.

[60] Roberts, A. P., Garboczi, E. J. Elastic moduli of model random three-dimensional closed-cell cellular solids. Acta Mater, 2001, 49(2), 189-197.

[61] Dawson, J. R., Shortall, J. B. The microstructure of rigid polyurethane foams. J Mater Sci, 1982, 17(1), 220-224.

[62] Saha, M. C., Mahfuz, H., Chakravarty, U. K., Uddin, M., Kabir, M. E., Jeelani, S. Effect of density, microstructure, and strain rate on compression behavior of polymeric foams. Materials Science and Engineering: A, 2005,406(1-2), 328-336.

[63] Walsh, J. B., Brace, W. F., England, A. W. Effect of porosity on compressibility of glass. J Am Ceram Soc, 1965, 48(12), 605-608.

[64] Kim, Y. W., Jin, Y. J., Chun, Y. S., Song, I. H., Kim, H. D. A simple pressing route to closed-cell microcellular ceramics. Scripta materialia, 2005, 53(8), 921-925.

[65] Meza, L. R., Phlipot, G. P., Portela, C. M., Maggi, A., Montemayor, L. C., Comella, A., Greer, J. R. Reexamining the mechanical property space of three-dimensional lattice architectures. Acta Mater, 2017 , $140,424-432$. 